\title{
DISCRETE SCAN STATISTICS GENERATED BY EXCHANGEABLE BINARY TRIALS
}

\author{
SERKAN ERYILMAZ, ${ }^{*}$ Izmir University of Economics
}

\begin{abstract}
Let $\left\{X_{i}\right\}_{i=1}^{n}$ be a sequence of random variables with two possible outcomes, denoted 0 and 1 . Define a random variable $S_{n, m}$ to be the maximum number of $1 \mathrm{~s}$ within any $m$ consecutive trials in $\left\{X_{i}\right\}_{i=1}^{n}$. The random variable $S_{n, m}$ is called a discrete scan statistic and has applications in many areas. In this paper we evaluate the distribution of discrete scan statistics when $\left\{X_{i}\right\}_{i=1}^{n}$ consists of exchangeable binary trials. We provide simple closed-form expressions for both conditional and unconditional distributions of $S_{n, m}$ for $2 m \geq n$. These results are also new for independent, identically distributed Bernoulli trials, which are a special case of exchangeable trials.
\end{abstract}

Keywords: Bernoulli sequence; exchangeable binary trial; reliability; scan statistics

2010 Mathematics Subject Classification: Primary 60E05; 62E15

\section{Introduction}

Scan statistics are used in diverse fields including epidemiology, molecular biology, reliability, and quality control. In the literature, discrete and continuous scan statistics in onedimensional as well as multidimensional cases have been defined and extensively studied. For excellent and comprehensive reviews of scans and their applications, we refer the reader to Glaz et al. (2001), and Balakrishnan and Koutras (2002).

Let $\left\{X_{i}\right\}_{i=1}^{n}$ be a sequence of random variables with two possible outcomes, denoted ' 0 ' (failure) and ' 1 ' (success). The discrete scan statistic of window size $m$ for the sequence $\left\{X_{i}\right\}_{i=1}^{n}$ is defined as

$$
S_{n, m}=\max \left\{\sum_{j=i}^{i+m-1} X_{j}: 1 \leq i \leq n-m+1\right\} .
$$

The main problem in this context is the evaluation of $\mathrm{P}\left\{S_{n, m}<k\right\}$ under various assumptions on $\left\{X_{i}\right\}_{i=1}^{n}$. An exact formula for $\mathrm{P}\left\{S_{n, m}<k\right\}$ is not available for arbitrary values of $n, m$, and $k$ even in the simplest case when the $\left\{X_{i}\right\}_{i=1}^{n}$ are independent and identically distributed (i.i.d.) Bernoulli trials. To the author's knowledge, the best formula currently available for $\mathrm{P}\left\{S_{n, m}<k\right\}$ exists only for the case $n=m L, L \geq 2$, when $\left\{X_{i}\right\}_{i=1}^{n}$ consists of i.i.d. Bernoulli trials (see Naus (1974)). Because of the difficulty in finding the exact value of $\mathrm{P}\left\{S_{n, m}<k\right\}$, accurate approximations and inequalities have been developed in the literature especially for the case when $\left\{X_{i}\right\}_{i=1}^{n}$ consists of independent elements. Some recent contributions on the topic, among others, are the works of Haiman (2007), Zhenkiu and Glaz (2008), and Inoue and Aki (2009).

Received 11 January 2010; revision received 29 June 2010.

* Current address: Department of Industrial Engineering, Atilim University, 06836 Incek, Ankara, Turkey.

Email address: seryilmaz@atilim.edu.tr 
In this paper we study $\mathrm{P}\left\{S_{n, m}<k\right\}$ for the case when $\left\{X_{i}\right\}_{i=1}^{n}$ consists of exchangeable dependent binary trials. It is worth mentioning that $\mathrm{P}\left\{S_{n, k}<k\right\}=\mathrm{P}\left\{L_{n}<k\right\}$, where $L_{n}$ is the length of the longest success run, which has recently been studied for exchangeable binary trials (see Eryılmaz and Demir (2007), Makri et al. (2007a), (2007b), Eryılmaz (2008a), (2008b) and Makri and Psillakis (2010)).

A finite set of binary random variables $\left\{X_{i}\right\}_{i=1}^{n}$ is said to be exchangeable if, for any $r(\leq n)$ and any vector $\left(x_{1}, \ldots, x_{r}\right)$, with $x_{i}=0,1, i=1, \ldots, r$,

$$
\mathrm{P}\left\{X_{\pi(1)}=x_{1}, \ldots, X_{\pi(r)}=x_{r}\right\}=\mathrm{P}\left\{X_{1}=x_{1}, \ldots, X_{r}=x_{r}\right\}
$$

for any permutation $\pi=(\pi(1), \ldots, \pi(r))$ of $\{1, \ldots, r\}$. For example, the probabilities of two sequences 10011 and 01101 are the same under exchangeability.

There might be various situations for studying scans for a sequence of exchangeable binary trials. One of the widely studied system models in reliability analysis is a linear consecutive $k$-within- $m$-out-of- $n$ : $F$ system, consisting of $n$ linearly ordered components such that the system fails if and only if there are $m$ consecutive components which include among them at least $k$ failed components (see, e.g. Kuo and Zuo (2003, pp. 407-410)). If $X_{i}$ denotes the state of the $i$ th component ( $X_{i}=1$ if the $i$ th component fails and $X_{i}=0$ if it works), then $\mathrm{P}\left\{S_{n, m}<k\right\}$ gives the reliability of the consecutive $k$-within- $m$-out-of- $n: F$ system. Let $T_{1}, \ldots, T_{n}$ denote the lifetimes of components, and define $X_{i}(t)=1$ if $T_{i} \leq t$ and $X_{i}(t)=0$ if $T_{i}>t, i=1, \ldots, n$. Then $X_{i}(t)$ represents the state of the $i$ th component at time $t$. If $T_{k, m: n}$ denotes the lifetime of the consecutive $k$-within- $m$-out-of- $n: F$ system then $\mathrm{P}\left\{T_{k, m: n}>t\right\}=\mathrm{P}\left\{S_{n, m}(t)<k\right\}$, where $S_{n, m}(t)$ is the scan statistic in $\left\{X_{i}(t)\right\}_{i=1}^{n}$. In most cases the lifetimes of components, $T_{1}, \ldots, T_{n}$, are dependent and have an exchangeable joint distribution. The exchangeability of the $T_{i} \mathrm{~s}$ implies the exchangeability of $\left\{X_{i}(t)\right\}_{i=1}^{n}$. Thus, the reliability evaluation of this particular system with exchangeable component lifetimes needs to include the study of the distribution of discrete scan statistics in an exchangeable sequence.

The paper is organized as follows. In Section 2 we present exact expressions for both conditional and unconditional distributions of $S_{n, m}$ for $2 m \geq n$ when $\left\{X_{i}\right\}_{i=1}^{n}$ consists of i.i.d. and exchangeable binary trials. In Section 3 we present some numerical results on the reliability of the abovementioned systems to illustrate the findings of the paper. The results included in this paper are not only an extension to exchangeable trials but also new for i.i.d. trials.

\section{Exact distributions}

Let $\left\{X_{i}\right\}_{i=1}^{n}$ be a sequence of exchangeable trials with two possible outcomes, denoted ' 0 ' and ' 1 ', and let $S_{n, n}=\sum_{i=1}^{n} X_{i}$. Using an inclusion and exclusion principle, George and Bowman (1995) proved that

$$
\mathrm{P}\left\{S_{n, n}=l\right\}=\left(\begin{array}{l}
n \\
l
\end{array}\right) p(l, n)=\left(\begin{array}{l}
n \\
l
\end{array}\right) \sum_{i=0}^{n-l}(-1)^{i}\left(\begin{array}{c}
n-l \\
i
\end{array}\right) \lambda_{l+i},
$$

where, for the subset $\left\{i_{1}, \ldots, i_{r}\right\}$ of $\{1, \ldots, n\}, r=1, \ldots, n$,

$$
\lambda_{r}=\mathrm{P}\left\{X_{i_{1}}=1, \ldots, X_{i_{r}}=1\right\}=\mathrm{P}\left\{X_{1}=1, \ldots, X_{r}=1\right\},
$$

and $\lambda_{0}=1$.

From (1) we have

$$
\mathrm{P}\left\{\sum_{j=1}^{s} X_{j}=l, X_{s+1}=x_{s+1}, \ldots, X_{n}=x_{n}\right\}=\left(\begin{array}{l}
s \\
l
\end{array}\right) p\left(l+\sum_{i=s+1}^{n} x_{i}, n\right) .
$$


It is clear that if $\lambda_{r}=\lambda_{1}^{r}$ for $r=1, \ldots, n$ then $\left\{X_{i}\right\}_{i=1}^{n}$ defines a Bernoulli sequence with success probability $p=\lambda_{1}$, and in this case $S_{n, n}$ is a binomial random variable.

Exact expressions for conditional and unconditional distributions of $S_{n, m}$ are available only for a restricted range of parameters when $\left\{X_{i}\right\}_{i=1}^{n}$ consists of i.i.d. Bernoulli trials. Saperstein (1972) obtained combinatorial formulae for the conditional distribution of $S_{n, m}$ given the number of successes $S_{n, n}=l$ for i.i.d. Bernoulli trials when $2 l \leq n+1$. Naus (1974) derived the following formula for i.i.d. Bernoulli trials when $n=m L, L \geq 2$ :

$$
\mathrm{P}(k ; m, n, l)=\mathrm{P}\left\{S_{n, m}<k \mid S_{n, n}=l\right\}=\frac{(m !)^{L}}{\left(\begin{array}{l}
n \\
l
\end{array}\right)} \sum_{\sigma \in S_{k}} \operatorname{det}\left|d_{i j}\right|,
$$

where

$$
\begin{aligned}
& d_{i j}=\frac{1}{c_{i j} !\left(m-c_{i j}\right) !}, \\
& c_{i j}= \begin{cases}(j-i) k-\sum_{r=1}^{j-1} n_{r}+n_{i} & \text { for } i<j, \\
(j-i) k+\sum_{r=j}^{i} n_{r} & \text { for } i \geq j,\end{cases}
\end{aligned}
$$

$n_{i}$ is the number of $1 \mathrm{~s}$ in the trials $(i-1) m+1, \ldots, i m, 1 \leq i \leq L$, and $S_{k}$ denotes the set of all partitions of $l$ into $L$ nonnegative integers each less than $k$.

Naus (1982) obtained the following simple expression for the unconditional distribution of $S_{2 m, m}$ for i.i.d. Bernoulli trials:

$$
\begin{aligned}
Q(k, m)= & \mathrm{P}\left\{S_{2 m, m}<k\right\} \\
= & F^{2}(k-1 ; m, p)-(k-1)\left(\begin{array}{c}
m \\
k
\end{array}\right) p^{k}(1-p)^{m-k} F(k-2 ; m, p) \\
& +m p\left(\begin{array}{c}
m \\
k
\end{array}\right) p^{k}(1-p)^{m-k} F(k-3 ; m-1, p),
\end{aligned}
$$

where $F(r ; s, p)=\sum_{i=0}^{r}\left(\begin{array}{l}s \\ i\end{array}\right) p^{i}(1-p)^{s-i}$.

We first obtain the unconditional distribution of $S_{n, m}$ for i.i.d. Bernoulli trials when $2 m \geq n$ in terms of $Q(k, m)$. The following simple combinatorial formula is more general and more efficient than the formulae given in Naus (1974), (1982).

Theorem 1. Let $\left\{X_{i}\right\}_{i=1}^{n}$ be a sequence of i.i.d. Bernoulli trials with success probability $p$. Then, for $2 m \geq n$,

$$
\begin{aligned}
P\left\{S_{n, m}<k\right\}=\sum_{s=0}^{\min (n-m, k-1)} \sum_{j=0}^{\min (2 m-n, k-s-1)} & \left(\begin{array}{c}
2 m-n \\
j
\end{array}\right) p^{j}(1-p)^{2 m-n-j} \\
& \times[Q(s+1, n-m)-Q(s, n-m)] .
\end{aligned}
$$

Proof. By the definition of $S_{n, m}$,

$$
\mathrm{P}\left\{S_{n, m}<k\right\}=\mathrm{P}\left\{\sum_{j=1}^{m} X_{j}<k, \sum_{j=2}^{m+1} X_{j}<k, \ldots, \sum_{j=n-m+1}^{n} X_{j}<k\right\} .
$$


For $2 m \geq n$,

$$
\begin{gathered}
\mathrm{P}\left\{S_{n, m}<k\right\}=\sum_{x_{1}, \ldots, x_{n-m}, x_{m+1}, \ldots, x_{n} \in\{0,1\}} \mathrm{P}\left\{\sum_{i=n-m+1}^{m} X_{i}<m^{*}, X_{1}=x_{1}, \ldots, X_{n-m}=x_{n-m},\right. \\
\left.X_{m+1}=x_{m+1}, \ldots, X_{n}=x_{n}\right\},
\end{gathered}
$$

where

$$
\begin{aligned}
m^{*} & =\min \left(k-x_{1}-\cdots-x_{n-m}, k-x_{2}-\cdots-x_{n-m}-x_{m+1}, \ldots, k-x_{m+1}-\cdots-x_{n}\right) \\
& =k-\max \left(x_{1}+\cdots+x_{n-m}, x_{2}+\cdots+x_{n-m}+x_{m+1}, \ldots, x_{m+1}+\cdots+x_{n}\right) .
\end{aligned}
$$

If $S_{2(n-m), n-m}^{*}$ denotes the scan statistic based on $X_{1}, \ldots, X_{n-m}, X_{m+1}, \ldots, X_{n}$ then

$$
\mathrm{P}\left\{S_{n, m}<k\right\}=\sum_{s=0}^{\min (n-m, k-1)} \mathrm{P}\left\{\sum_{i=n-m+1}^{m} X_{i}<k-s, S_{2(n-m), n-m}^{*}=s\right\} .
$$

Because $\left\{X_{i}\right\}_{i=1}^{n}$ consists of i.i.d. trials, $\sum_{i=n-m+1}^{n} X_{i}$ and $S_{2(n-m), n-m}^{*}$ are independent. Thus, the proof is completed by observing that

$$
\mathrm{P}\left\{\sum_{i=n-m+1}^{m} X_{i}=j\right\}=\left(\begin{array}{c}
2 m-n \\
j
\end{array}\right) p^{j}(1-p)^{2 m-n-j}
$$

and

$$
\begin{aligned}
\mathrm{P}\left\{S_{2(n-m), n-m}^{*}=s\right\} & =\mathrm{P}\left\{S_{2(n-m), n-m}^{*}<s+1\right\}-\mathrm{P}\left\{S_{2(n-m), n-m}^{*}<s\right\} \\
& =Q(s+1, n-m)-Q(s, n-m) .
\end{aligned}
$$

Corollary 1. For $m=k$ in Theorem 1 , we obtain

$$
\mathrm{P}\left\{S_{n, k}<k\right\}=\mathrm{P}\left\{L_{n}<k\right\}=1-(n-k+1) p^{k}+(n-k) p^{k+1}
$$

for $2 k \geq n$, which corresponds to that found in Tong (1985).

Remark 1. Since the exchangeability implies that all sequences with the same number of successes are equally likely, it is evident that $\mathrm{P}\left\{S_{n, m}<k \mid S_{n, n}=l\right\}$ does not depend on the particular distribution of $\left(X_{1}, \ldots, X_{n}\right)$ when the $\left\{X_{i}\right\}_{i=1}^{n}$ are exchangeable (and, in particular, i.i.d.). Thus,

$$
\mathrm{P}\left\{S_{n, m}<k \mid S_{n, n}=l\right\}=\left(\begin{array}{l}
n \\
l
\end{array}\right)^{-1} N(l, k, m, n),
$$

where $N(l, k, m, n)$ is the number of binary sequences of length $n$ with $l$ successes such that there are less than $k$ successes among any consecutive $m$ trials.

Theorem 2. Let $\left\{X_{i}\right\}_{i=1}^{n}$ be a sequence of exchangeable binary trials. Then, for $2 m \geq n$,

$$
\begin{aligned}
& \mathrm{P}\left\{S_{n, m}<k \mid S_{n, n}=l\right\} \\
&=\left(\begin{array}{l}
n \\
l
\end{array}\right) \sum_{s=0}^{-1} \sum_{j=\max (0, l-2(n-m))}^{\min (n-m, k-1)}\left(\begin{array}{c}
2 m-n \\
j
\end{array}\right)\left(\begin{array}{c}
2(n-m) \\
l-j
\end{array}\right) \\
& \times\left[\begin{array}{l}
\mathrm{P}(s+1 ; n-m, 2(n-m), l-j) \\
-\mathrm{P}(s ; n-m, 2(n-m), l-j)] .
\end{array}\right.
\end{aligned}
$$


Proof. Using the notation given in the proof of Theorem 1, we have

$$
\begin{aligned}
\mathrm{P}\left\{S_{n, m}<k \mid S_{n, n}=l\right\} & \\
= & \sum_{s=0}^{\min (n-m, k-1)} \mathrm{P}\left\{\sum_{i=n-m+1}^{m} X_{i}<k-s, S_{2(n-m), n-m}^{*}=s \mid S_{n, n}=l\right\} .
\end{aligned}
$$

Because $\mathrm{P}\left\{S_{n, m}<k \mid S_{n, n}=l\right\}$ is identical for both i.i.d. and exchangeable trials,

$$
\begin{aligned}
\mathrm{P}\left\{S_{n, m}<k \mid S_{n, n}=l\right\} & \\
=\frac{1}{\mathrm{P}\left\{S_{n, n}=l\right\}} & \\
& \times \sum_{s=0}^{\min (n-m, k-1) \min (l, 2 m-n, k-s-1)} \\
& \mathrm{P}\left\{\sum_{j=0}^{m} X_{i=n-m+1}=j\right\} \\
& \times \mathrm{P}\left\{S_{2(n-m), n-m}^{*}=s \mid S_{2(n-m), 2(n-m)}^{*}=l-j\right\} \\
& \times \mathrm{P}\left\{S_{2(n-m), 2(n-m)}^{*}=l-j\right\} .
\end{aligned}
$$

Thus, the proof follows from

$$
\frac{\mathrm{P}\left\{\sum_{i=n-m+1}^{m} X_{i}=j\right\} \mathrm{P}\left\{S_{2(n-m), 2(n-m)}^{*}=l-j\right\}}{\mathrm{P}\left\{S_{n, n}=l\right\}}=\left(\begin{array}{c}
2 m-n \\
j
\end{array}\right)\left(\begin{array}{c}
2(n-m) \\
l-j
\end{array}\right)\left(\begin{array}{l}
n \\
l
\end{array}\right)^{-1},
$$

and using (3) for $\mathrm{P}\left\{S_{2(n-m), n-m}^{*}=s \mid S_{2(n-m), 2(n-m)}^{*}=l-j\right\}$ when $L=2$.

The proof of the following result immediately follows by conditioning on the number of successes and using the observation given in Remark 1.

Theorem 3. Let $\left\{X_{i}\right\}_{i=1}^{n}$ be a sequence of exchangeable binary trials with $\lambda_{r}=\mathrm{P}\left\{X_{i_{1}}=\right.$ $\left.1, \ldots, X_{i_{r}}=1\right\}, r=1, \ldots, n$. Then, for $2 m \geq n$,

$$
\begin{aligned}
& \mathrm{P}\left\{S_{n, m}<k\right\}=\sum_{l=0}^{n} \sum_{s=0}^{\min (n-m, k-1)} \sum_{j=\max (0, l-2(n-m))}^{\min (l, 2 m-n, k-s-1)}\left(\begin{array}{c}
2 m-n \\
j
\end{array}\right)\left(\begin{array}{c}
2(n-m) \\
l-j
\end{array}\right) \\
& \times[\mathrm{P}(s+1 ; n-m, 2(n-m), l-j) \\
& -\mathrm{P}(s ; n-m, 2(n-m), l-j)] p(l, n),
\end{aligned}
$$

where

$$
p(l, n)=\sum_{i=0}^{n-l}(-1)^{i}\left(\begin{array}{c}
n-l \\
i
\end{array}\right) \lambda_{l+i} .
$$

Remark 2. From Theorems 1, 2, and 3, we observe that the conditional and unconditional distributions of $S_{n, m}$ for $2 m=n$ are enough to obtain the corresponding distributions of $S_{n, m}$ for $2 m \geq n$ when $\left\{X_{i}\right\}_{i=1}^{n}$ is a sequence of i.i.d. or exchangeable binary trials.

\section{Application to reliability}

Since the reliability of the consecutive $k$-within- $m$-out-of- $n: F$ system is defined by the probability $R_{k, m: n}=\mathrm{P}\left\{S_{n, m}<k\right\}$, the findings of this paper can be effectively used to evaluate 
TABLE 1: Exact reliability of the consecutive $k$-within- $m$-out-of- $n: F$ system with i.i.d. components.

\begin{tabular}{cccc}
\hline$n$ & $m$ & $k$ & $R_{k, m: n}$ \\
\hline 10 & 5 & 3 & 0.9707 \\
& 5 & 4 & 0.9980 \\
& 6 & 3 & 0.9596 \\
12 & 6 & 3 & 0.9479 \\
& 7 & 3 & 0.9339 \\
& 7 & 4 & 0.9911 \\
15 & 8 & 3 & 0.8933 \\
& 8 & 4 & 0.9817 \\
& 8 & 5 & 0.9980 \\
& 10 & 3 & 0.8613 \\
& 10 & 4 & 0.9689 \\
& 10 & 6 & 0.9995 \\
20 & 10 & 4 & 0.9521 \\
& 12 & 4 & 0.9304 \\
30 & 15 & 4 & 0.8247 \\
& 15 & 5 & 0.9484 \\
40 & 20 & 5 & 0.8498 \\
& 21 & 5 & 0.8346 \\
\hline
\end{tabular}

the reliability of this system. It is worth mentioning that this model involves consecutive $k$-outof- $n$ : $F$ and $k$-out-of- $n: F$ systems for $m=k$ and $m=n$, respectively. The result provided in Theorem 1 is the simplest formula that has been obtained in the literature for computing $R_{k, m: n}$ when $2 m \geq n$. For illustrative purposes, in Table 1 we present some values of $R_{k, m: n}$, assuming that the system components are independent and have the same failure probability, $p=0.1$.

Using Theorem 3, we can compute the reliability of the consecutive $k$-within- $m$-out-of- $n: F$ system consisting of exchangeable components. Let the components have an exchangeable Lomax distribution with joint survival function

$$
\bar{F}\left(t_{1}, \ldots, t_{n}\right)=\mathrm{P}\left\{T_{1}>t, \ldots, T_{n}>t\right\}=\left(1+\sum_{i=1}^{n} t_{i}\right)^{-\alpha}
$$

for $\alpha>0$ and $t_{i}>0, i=1, \ldots, n$. Then

$$
\lambda_{r}=\mathrm{P}\left\{T_{1}>t, \ldots, T_{r}>t\right\}=(1+r t)^{-\alpha}
$$

for $r=1, \ldots, n$. The survival function of the consecutive $k$-within- $m$-out-of- $n: F$ system is given by

$$
R_{k, m: n}(t)=\mathrm{P}\left\{T_{k, m: n}>t\right\}=\mathrm{P}\left\{S_{n, m}(t)<k\right\}
$$

and can be computed exactly using Theorem 3 when $2 m \geq n$.

In Table 2 we present some values of $R_{k, m: n}(0.1)$ for various values of $k, m$, and $n(2 m \geq n)$ when $\alpha=1$.

The survival function of any coherent system with lifetime $T=\phi\left(T_{1}, \ldots, T_{n}\right)$ and exchangeable component lifetimes $T_{1}, \ldots, T_{n}$ having an absolutely continuous joint distribution 
TABLE 2: Exact reliability of the consecutive $k$-within- $m$-out-of- $n: F$ system with exchangeable components.

\begin{tabular}{cccc}
\hline$n$ & $m$ & $k$ & $R_{k, m: n}(0.1)$ \\
\hline 10 & 5 & 3 & 0.9425 \\
& 5 & 4 & 0.9879 \\
& 6 & 3 & 0.9283 \\
12 & 6 & 3 & 0.9130 \\
& 7 & 3 & 0.8985 \\
& 7 & 4 & 0.9657 \\
15 & 8 & 3 & 0.8594 \\
& 8 & 4 & 0.9441 \\
& 8 & 5 & 0.9812 \\
& 10 & 3 & 0.8347 \\
& 10 & 4 & 0.9237 \\
& 10 & 6 & 0.9882 \\
20 & 10 & 4 & 0.8985 \\
& 12 & 4 & 0.8760 \\
30 & 15 & 4 & 0.7913 \\
& 15 & 5 & 0.8769 \\
40 & 20 & 5 & 0.7949 \\
& 21 & 5 & 0.7862 \\
\hline
\end{tabular}

can be written as a mixture of survival functions of order statistics, i.e.

$$
\mathrm{P}\{T>t\}=\sum_{i=1}^{n} p_{i} \mathrm{P}\left\{T_{i: n}>t\right\}
$$

(see Samaniego (1985), Kochar et al. (1999), and Navarro and Rychlik (2007)). Here $p_{i}$ is the probability that the system fails upon the occurrence of the $i$ th component failure, i.e. $p_{i}=\mathrm{P}\left\{T=T_{i: n}\right\}$. The vector $\boldsymbol{p}=\left(p_{1}, \ldots, p_{n}\right)$ is called the system signature. The system signature has been found to be a useful tool in a variety of applications, including the evaluation of the reliability characteristics of systems and the comparison of the performance of competing systems. For the details of the notion of a signature and its applications in reliability engineering, the reader is referred to the excellent monograph in Samaniego (2007).

The signature of a system consisting of exchangeable components can be computed by finding the number of path sets of the system with exactly $i$ working components. Let $r_{i}(n)$ denote the number of path sets of the system with exactly $i$ working components. Define

$$
a_{i}(n)=\frac{r_{i}(n)}{\left(\begin{array}{c}
n \\
i
\end{array}\right)}, \quad i=1,2, \ldots, n .
$$

Then the quantities

$$
p_{i}=a_{n-i+1}(n)-a_{n-i}(n), \quad i=1,2, \ldots, n,
$$

give the signature of a system, where $a_{0}(n)=0$ (see, e.g. Boland (2001)).

The formula currently available for the signature of the consecutive $k$-within- $m$-out-of- $n: F$ system exists only for the case $k=2$ (see Eryılmaz (2010)). Using Theorem 2, we can obtain 
TABLE 3: Signatures of consecutive $k$-within- $m$-out-of- $n: F$ systems.

\begin{tabular}{cccc}
\hline$n$ & $m$ & $k$ & Signature \\
\hline 5 & 3 & 2 & $(0,0.7,0.3,0,0)$ \\
& 3 & 3 & $(0,0,0.3,0.5,0.2)$ \\
& 4 & 2 & $(0,0.9,0.1,0,0)$ \\
& 4 & 3 & $(0,0,0.7,0.3,0)$ \\
10 & 5 & 3 & $(0,0,0.3333,0.4286,0.2381,0,0,0,0,0)$ \\
& 5 & 5 & $(0,0,0,0,0.0238,0.0952,0.2143,0.3333,0.3333,0)$ \\
& 6 & 3 & $(0,0,0.5,0.4048,0.0952,0,0,0,0,0)$ \\
& 6 & 4 & $(0,0,0,0.2619,0.4206,0.2698,0.0476,0,0,0)$ \\
\hline
\end{tabular}

the signature of this system for $2 m \geq n$ and an arbitrary value of $k$. If $N(l, k, m, n)$ denotes the number of ways of arranging a total of $n$ components with $l$ failed components and $n-l$ working components in a line such that less than $k$ components are failed among any consecutive $m$ components, then

$$
\mathrm{P}\left\{S_{n, m}<k \mid S_{n, n}=l\right\}=\left(\begin{array}{l}
n \\
l
\end{array}\right)^{-1} N(l, k, m, n) .
$$

By Theorem 2, we obtain

$$
\begin{aligned}
N(l, k, m, n)=\sum_{s=0}^{\min (n-m, k-1)} \sum_{j=\max (0, l-2(n-m))}^{\min (l, 2 m-n, k-s-1)}\left(\begin{array}{c}
2 m-n \\
j
\end{array}\right) \\
\times[N(l-j, s+1, n-m, 2(n-m)) \\
-N(l-j, s, n-m, 2(n-m))]
\end{aligned}
$$

for $2 m \geq n$.

By virtue of (4) and (5), the signature of the consecutive $k$-within- $m$-out-of- $n: F$ system for $2 m \geq n$ can be computed from

$$
p_{i}=\frac{N(i-1, k, m, n)}{\left(\begin{array}{c}
n \\
n-i+1
\end{array}\right)}-\frac{N(i, k, m, n)}{\left(\begin{array}{c}
n \\
n-i
\end{array}\right)}
$$

for $i \geq k$, and $p_{i}=0$ for $i<k$.

In Table 3 we present signatures of various consecutive $k$-within- $m$-out-of- $n: F$ systems for selected values of $n, m$, and $k$.

\section{Acknowledgement}

The author is grateful to the anonymous referee, whose meticulous reading helped improve the quality of the paper.

\section{References}

Balakrishnan, N. and Koutras, M. V. (2002). Runs and Scans with Applications. John Wiley, New York. Boland, P. J. (2001). Signatures of indirect majority systems. J. Appl. Prob. 38, 597-603.

Eryilmaz, S. (2008a). Distribution of runs in a sequence of exchangeable multi-state trials. Statist. Prob. Lett. 78, 1505-1513.

Eryilmaz, S. (2008b). Run statistics defined on the multicolor urn model. J. Appl. Prob. 45, 1007-1023. 
Eryilmaz, S. (2010). Mixture representations for the reliability of consecutive- $k$ systems. Math. Comput. Modelling 51, 405-412.

Eryilmaz, S. And Demir, S. (2007). Success runs in a sequence of exchangeable binary trials. J. Statist. Planning Infer. 137, 2954-2963.

George, E. O. And Bowman, D. (1995). A full likelihood procedure for analysing exchangeable binary data, Biometrics $\mathbf{5 1 , 5 1 2 - 5 2 3 .}$

Glaz, J., Naus, J. and Wallanstein, S. (2001). Scan Statistics, Springer, New York.

HaIman, G. (2007). Estimating the distribution of one-dimensional discrete scan statistics viewed as extremes of 1-dependent stationary sequences. J. Statist. Planning Infer. 137, 821-828.

InOUe, K. AND AKI, S. (2009). Distributions of runs and scans on higher-order Markov trees. Commun. Statist. Theory Meth. 38, 621-641.

Kochar, S., Mukerjee, H. and Samaniego F. J. (1999). The 'signature' of a coherent system and its application to comparison among systems. Naval Res. Logistics 46, 507-523.

Kuo, W. And Zuo, M. J. (2003). Optimal Reliability Modeling: Principles and Applications. John Wiley, Hoboken, NJ.

MaKri, F. S. AND Psillakis, Z. M. (2010). On success runs of length exceeded a threshold. To appear in Methodology Comput. Appl. Prob.

Makri, F. S., Philippou, A. N. And Psillakis, Z. M. (2007a). Shortest and longest length of success runs in binary sequences. J. Statist. Planning Infer. 137, 2226-2239.

Makri, F. S., Philippou, A. N. AND Psillakis, Z. M. (2007b). Success run statistics defined on an urn model, $A d v$. Appl. Prob. 39, 991-1019.

NaUS, J. (1974). Probabilities for a generalized birthday problem. J. Amer. Statist. Assoc. 69, 810-815.

NaUs, J. I. (1982). Approximations for distributions of scan statistics. J. Amer. Statist. Assoc. 77, 177-183.

NAVARro, J. AND Rychlik, T. (2007). Reliability and expectation bounds for coherent systems with exchangeable components. J. Multivariate Anal. 98, 102-113.

Samaniego, F. J. (1985). On closure of the IFR class under formation of coherent systems. IEEE Trans. Reliab. 34, 69-72.

SAMANIEGo, F. J. (2007). System signatures and their applications in engineering reliability. Springer, New York.

SAPERSTEIn, B. (1972). The generalized birthday problem. J. Amer. Statist. Assoc. 67, 425--428.

TonG, Y. L. (1985). A rearrangement inequality for the longest run, with an application to network reliability. J. Appl. Prob. 22, 386-393.

ZhenkiU, Z. ANd GlaZ, J. (2008). Bayesian variable window scan statistics. J. Statist. Planning Infer. 138, 3561-3567. 